\title{
The effect of texture on tactually perceived length ${ }^{1}$
}

\author{
DAVID A. CORSINI, ${ }^{2}$ UNIVERSITY OF GEORGIA \\ HERBERT L. PICK, JR., UNIVERSITY OF MINNESOTA
}

\begin{abstract}
A curvilinear relationship between texture and perceived length was investigated in. two experiments. In Experiment 1 the perceived lengths of four grades of emery cloth and an untextured stimulus were compared in a forced-choice procedure. Generally, as predicted, fine-texture stimuli were perceived as longer than coarse-textured stimuli. Experiment 2 attempted to modify the biasing effect of texture by employing preliminary training with fine or coarse textures. Although unsuccessful in modifying the bias, the curvilinear relationship between texture and perceived length was again supported. Some implications for constructing teaching materials for the blind are discussed.
\end{abstract}

This report presents the results of two investigations concerned with the effect that texture differences have upon tactually perceived length. These studies explore, in a more systematic manner, a perceptual phenomenon originally suggested by Soviet investigators (Lomov \& Vekker, 1960) ${ }^{3}$ and are initial attempts to find stimulus materials that could be used to present information to the blind. Presently, there are numerous problems in constructing tactile material for the blind to convey the information provided for the sighted by maps, charts, and graphs (Schiff, Kanfer, \& Mosak, 1966; Sherman, 1965). For example, there has been found no phenomenon in tactual perception that allows for the representation of depth in the two-dimensional plane, as does linear perspective in visual perception. Part of the problem in providing representational material for the blind is to find material that allows for numerous values to be easily discriminated tactually. Several reports (Nolan \& Morris, 1960; Stevens \& Harris, 1962) have indicated that the different textures of the various grades of emery cloth do provide stimuli that are both easily discriminated and reliably ordered on a dimension of roughness. In order to use most efficiently this dimension to convey information to the blind, it is important to increase our understanding of how these particular materials affect different aspects of tactual performance.

In several pilot studies blindfolded adult Ss were asked to make tactual length judgments of differently textured stimuli (different grades of emery cloth) among which there were no objective differences in length. Although several different procedures and variations in the stimulus materials were used, it was consistently found that stimuli constructed of relatively fine-textured emery cloth were judged to be somewhat longer than a nontextured standard, whereas stimuli constructed of relatively coarse-textured emery cloth were judged to be shorter than the standard. Of these two findings the latter was more reliable. The consistency of these findings led to a tentative hypothesis that specified a curvilinear relationship between texture and perceived length. The hypothesis states that as texture varies from fine to coarse, length will be first overestimated and then underestimated.

The first study had two primary purposes: (a) to substantiate the curvilinear relationship between tex ture and perceived length; and (b) to determine the degree of this effect by observing the facilitating and interfering effects of texture in the discrimination of real length differences. The second study attempted to modify the biasing effect of texture by giving Ss preliminary discrimination training which was either consonant or dissonant with the biasing effect of texture.

\section{EXPERIMENT 1 \\ Method}

\section{Stimuli}

The stimili were constructed of material representing five different textures-oaktag paper and four grades of $3 \mathrm{M}$ Elek-tro-cut Cloth (aluminum oxide). The four grades of emery cloth had grit values of $320,120,50$, and 24 . Each of the five stimulus materials was represented by five different lengths: $7,7-3 / 16,7-6 / 16,7-9 / 16$, and $7-12 / 16$ in. This yielded a total of 25 different stimuli. Each stimulus was 4 in. in width and was mounted individually on a piece of heavy cardboard. Each stimulus was mounted 3 in. from the bottom of the cardboard and $3 \mathrm{in}$. from the left-hand edge in order that length differences extended only in the right-hand plane. The cardboard on which the stimuli were mounted was placed into a Masonite frame in order that stimuli were always in the same location from trial to trial.

\section{Subjects}

Ten male sighted undergraduate students were paid for completion of the six sessions of the experiment. Two Ss were replaced after the first session since they had demonstrated an aversion to feeling the textured stimuli.

\section{General}

Stimuli were presented successively in pairs and the blindfolded $\mathrm{S}$ was asked to make a judgment as to which of the two stimuli felt longer. A forced-choice procedure was used. Each S made 300 forced-choice judgments over a period of six sessions. The 300 judgments were chosen to meet four criteria:

(1) A complete series of paired comparisons was run using the 7 -in. value of each of the five textures. Each $\mathbf{S}$ made five judgments for each of the 10 possible pairs yielding 50 judgments per $S$. This aspect of the design was included to substantiate the curvilinear relationship between texture and perceived length which had been observed in the pilot studies.

(2) A complete series of paired comparisons was run for the five different lengths of the oaktag paper. Each $S$ made four judgments for each of the 10 possible pairs yielding a total of 40 judgments. This aspect of the design was included to yield an estimate of the Ss' ability for tactual length discrimination unaffected by differences in texture.

(3) All five length values of each of the four textured stimuli were compared with each of the five length values of the oaktag paper. Each of the resulting 100 pairs was judged twice, yielding a total of 200 judgments. These pairings provided cases in which the textured stimuli were objectively longer than the paper standard by values of $3 / 16,6 / 16,9 / 16$, and $12 / 16$ in. Conversely, it provided pairings in which the textured stimuli were objectively shorter than the paper standard by the same values. By comparing the Ss' ability to discriminate these length differences in which texture was involved with their ability to discriminate length when no texture differences were involved, it would be possible to evaluate the magnitude and the direction of the length bias attributable to the different textures.

The reasoning is as follows: The curvilinear hypothesis, which says that the length of fine-textured stimuli are overestimated and the length of coarse-textured stimuli underestimated when compared to a paper standard, would 
predict discrimination of length to be superior under two stimulus conditions and inferior under two other conditions. Superior discrimination would be predicted when the length bias of the texture is in the direction of the objective length differences. This can occur in two ways: (a) when fine-textured stimuli are compared with an oaktag standard and the textured stimulus is objectively longer; and (b) when coarse-textured stimuli are compared with a paper standard and the textured stimulus is objectively shorter. Inferior discrimination would be predicted when the length bias is in a direction opposite to the objective length differences. This also can occur in two ways: (a) when fine-textured stimuli are compared with a paper standard and the textured stimulus is objectively shorter; and (b) when coarse-textured stimuli are compared with a paper standard and the textured stimulus is objectively longer. Thus, this aspect of the design was included to evaluate whether the length bias attributable to texture would interact with length discrimination-facilitating discrimination under some circumstances and hindering it under others.

(4) Each of the 7-in. values was compared with itself on two occasions yielding a total of 10 judgments. This aspect of the design was to check for a bias in the order of presentation of stimuli.

\section{Procedure}

The $\mathrm{S}$ was blindfolded and given the following instructions: "In this experiment I have strips of sandpaper and regular paper mounted on cardboard. I am going to ask you to feel two of the strips, one at a time. After you have felt both strips, I want you to tell me which one felt longer. I am more interested in your immediate impression of length than in what you would say after long deliberation. Use one hand and feel along the strip with as many fingers as you wish; but don't measure it by spreading out your fingers. While you should be careful, don't take too much time with any one of the pairs because there are quite a few pairs." Each $S$ was run in six sessions, making 50 judgments during each session. The order of presentation within pairs of stimuli was counterbalanced to decrease the chance of order effect. Judgments from the four design aspects of the experiment were spread over the six sessions to prevent confounding.

\section{Results}

The curvilinear relationship between texture and perceived length, which had been observed in preliminary studies, and upon which the hypotheses of the present study were based, was substantiated in two ways. First, a paired-comparison model (Woodworth \& Schlosberg, 1960) was constructed from those judgments involved in the complete paired comparison of the 7-in. value of the four textures and the oaktag paper. Figure 1 shows that the two coarser textures (50 and 24) are perceived as shorter than the oaktag paper while the two finer textures (320 and 120) are perceived as longer. Closer inspection reveals that the 120 emery cloth is perceived to be about equal to the oaktag paper while the 320 emery cloth is perceived as longer and the two coarser grades as shorter. Since, as texture goes from relatively fine to relatively coarse, length is first overestimated and then underestimated, the hypothesized curvilinear relationship between texture and length is upheld. Secondly, the reliability of this relationship was assessed by an analysis of variance performed on judgment scores made on trials on which the different textures were compared with oaktag paper and there were no objective length differences between the two stimuli. The objective length of the two stimuli being compared on a particular trial in these cases was equal but varied from 7 to $7-12 / 16$ in. for different pairs. For each texture each $S$ made 13 forced-choice judgments of this type. The analysis of variance was performed

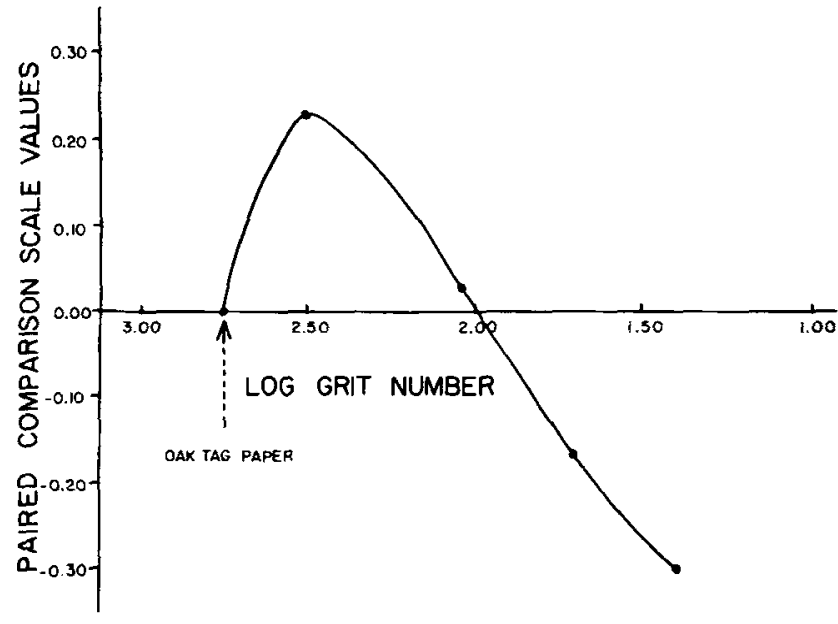

Fig. 1. Relation between scale values determined by paired comparison judgments and roughness of emery cloth. (Position of oaktag paper on abscissa determined by magnitude estimation rating.)

on the number of times the textured stimulus was chosen as longer than the oaktag paper in these 13 cases.

The analysis revealed a significant effect for the type of sandpaper $(F=7.96$, df $=3 / 27, p<.001)$. A Newman-Keuls (Winer, 1962) test of the difference between ordered means indicated that the 320 texture did not differ significantly from the 120 texture but that both of these were significantly different from the 50 and 24 textures, which in turn did not differ from each other. These results indicate that when the different textures were compared with paper and there were no objective differences between them, the two finer textures were perceived as longer significantly more often than were the two coarser textures. These results are also in line with the curvilinear relationship between texture and perceived length.

The above results are further clarified when the mean number of times the textured stimuli were chosen over the oaktag paper is compared with what would be expected by chance (6.5). T tests, using the MSE as the best estimate of variance (Lindquist, 1953), revealed that the $320(8.0)$ and 120 (7.5) textures were chosen as longer significantly more often than would be expected by chance $(t \geqslant 3.12$, df $=40$, $p<.005)$ and the $50(4.9)$ and $24(4.4)$ textures were chosen as longer significantly less often than would be expected by chance $(t \geqslant 4.98$, df $=40, p<.005)$.

The second aspect of the data analysis was concerned with the ability of the Ss to discriminate real length differences when unaffected by differences in texture. A complete paired comparison was performed for the five lengths of the oaktag paper. Table 1, in the column headed "untextured," shows the per cent of correct discrimination of length for the four length differences. Discrimination of length differences, when

Table 1

Mean Per Cent Accuracy of Length Discrimination (see text for explanation). Fine Texture $=320+120$ Emery Cloth;

Coarse Texture $=50+24$ Emery Cloth $;$ Untextured = Oaktag Paper.

\begin{tabular}{lllcll}
\hline $\begin{array}{l}\text { Length } \\
\text { Difference }\end{array}$ & $\begin{array}{l}\text { Fine } \\
\text { texture/ } \\
\text { longer }\end{array}$ & $\begin{array}{l}\text { Coarse } \\
\text { texture/ } \\
\text { longer }\end{array}$ & \multicolumn{2}{c}{$\begin{array}{l}\text { Fine } \\
\text { texture/ } \\
\text { untextured }\end{array}$} & $\begin{array}{l}\text { Coarse } \\
\text { texture/ } \\
\text { shorter }\end{array}$ \\
\hline $12 / 16$ in. & 87.5 & 65 & 90 & 87.5 & 90 \\
$9 / 16$ in. & 75.5 & 71 & 82 & 70 & 90.5 \\
$6 / 16$ in. & 70 & 54 & 69 & 66 & 65 \\
$3 / 16$ in. & 59 & 50.5 & 68 & 55 & 63.5 \\
\hline
\end{tabular}


unaffected by texture, was above $50 \%$ for all length differences used in this study. The Ss were able to discriminate the smallest difference, $3 / 16$ in., $68 \%$ of the time. These values for the Ss' ability to discriminate untextured length will be used to evaluate the hypotheses concerned with the effect of texture upon length discrimination.

The third aspect of the data analysis evaluated the hypothesis that differences in texture could mask or enhance real length differences depending on which textures were added to which lengths. The reasoning behind this hypothesis is based on the previous results which found that smooth textures seem longer than they are while coarse textures seem shorter. Thus, for example, if one wanted to make sure that a 7-in. stimulus would be judged shorter than a 7-3/16-in. stimulus, it is reasonable to assert that the 7 -in. stimulus should be made of coarse texture and the 7-3/16-in. stimulus made of smooth texture. While the present study was not designed to test length discrimination when both length and texture varied at one time, examination of the hypothesis concerned with the facilitating and interfering effects of texture upon discrimination can be examined in two ways.

Originally, it had been expected that the facilitating and interfering effects of texture could be demonstrated by comparing the accuracy of discrimination when texture was involved with the accuracy of discrimination when no texture was involved. Thus, from Table 1, it was expected that accuracy for "fine texture/longer" and "coarse texture/ shorter" would be better than the "untextured" accuracy; while the "coarse texture/longer" and "fine texture/shorter" would be worse than the "untextured" accuracy. Inspection of Table 1 shows that the expectations for interference, but not for facilitation, were upheld. However, it is possible that there was another factor, besides the bias attributable to texture, which may have been more important in discriminations involving a textured and untextured stimulus. Adding texture to the length discrimination may be equivalent to adding distraction or noise and have the overall effect of in terfering with effective discrimination. Even though facilitation was found only in cases for which it had been predicted (i.e., in no cases for which interference had been predicted) and almost all the predictions for inferior performance were supported, these results were not considered to constitute strong support for the biasing effect of texture. Stronger support is derived when the performance with the different textures are compared with each other rather than with the performance for the untextured comparisons.

To evaluate the performance with the different textures a repeated measures analysis of variance was performed on the per cent of correct responses on trials involving a textured and an oaktag stimulus. For all these pairs there were objective length differences. The specific predictions were: (a) When the smooth textures (320 and 120) are paired with oaktag papers that are shorter, length discrimination should be better than when smooth textures are paired with oaktag papers which are longer. (b) The opposite would be the case with the rough textures. When coarse textures (50 and 24) are paired with oaktag papers which are shorter, accurate length discrimination should be worse than when coarse textures are paired with oaktag papers which are longer.

Because Ss made different numbers of judgments for each of the objective length differences, the per cent of correct responses was used as the dependent measure. For each texture each $S$ made 16 judgments involving a 3/16-in. difference, 12 involving a $6 / 16$-in. difference, 8 involving a 9/16-in. difference, and 4 involving a 12/16-in. difference. Since different predictions were made depending upon whether it was the textured or oaktag paper which was the objectively longer stimulus, eight lengths were considered in the analysis-four in which the oaktag paper exceeded the textured stimulus and four in which the textured stimulus exceeded the oaktag paper. Thus, a 4 (Texture) by 8 (Length) repeated measures analysis of variance was performed.

This analysis showed a significant main effect for length ( $F=7.64, \quad \mathrm{df}=7 / 310, \quad \mathrm{p}<.01)$ which indicates that as objective length between stimuli increased, the per cent of correct responses also increased. No significant main effect was shown for the different textures. Since the predictions made for cases in which the textured stimulus was the objectively longer were opposite to those made for the case when the oaktag paper was the objectively longer, this result is not surprising. The interaction between texture and length was highly significant $(F=4.06, d f=21 / 310, p<.01)$. This significant interaction, was necessary to test the hypothesis that texture would affect length discrimination.

In order to make the interaction more easily understood, the following discussion will refer back to Table 1 where the mean per cent accuracy for the two fine textures and the two coarse textures are averaged. The interaction can be examined in two ways: (a) by comparing cases in which the textured stimuli were longer with cases in which the textured stimuli were shorter for each texture separately; and (b) by comparing between the two textures when the textured stimuli were longer and again for cases in which the textured stimuli were shorter.

From Table 1 it can be seen that in almost all cases the expectations from the curvilinear hypothesis were upheld: (a) when textured stimuli were longer, discrimination was better with the fine textures, and for the fine textures, discrimination was better when they were objectively longer than when they were objectively shorter; (b) when the textured stimuli were shorter, discrimination was better with the coarse textures, and for the coarse textures, discrimination was better when they were objectively shorter than when they were objectively longer.

\section{EXPERIMENT 2}

Experiment 1 supported the general hypothesis that, when compared with an untextured standard, lengths of fine texture are perceived as longer while lengths of coarse texture are perceived as shorter. Experiment 2 investigates whether the bias attributable to the different textures could be accentuated or attenuated by giving Ss preliminary training with different sandpaper stimuli.

\section{Subjects}

\section{Method}

Fifty undergraduate male $S s$ were used in this experiment-ten in each of five groups. Participation by $S$ fulfilled a requirement for an introductory course in psychology.

\section{Stimuli}

The stimuli were constructed from two grades of emery cloth and the untextured oaktag paper. The emery cloths had grit numbers of 320 (fine texture) and 50 (coarse texture). There were seven lengths of the three stimuli: $7,7-1 / 16$, $7-2 / 16,7-3 / 16,7-4 / 16,7-6 / 16$, and $7-8 / 16$ in. The stimuli were mounted on cardboard in the same manner as described in Experiment 1.

\section{General}

The four experimental groups received 24 trials of pretraining in length discrimination comparing one texture with the oaktag paper. The Ss were given information feedback as to the correctness of their performance on these 24 trials. Two experimental groups received pretraining with the stimuli arranged such that the bias attributable to texture was in the direction of the objective length differences: For Group 1 fine-textured stimuli were compared to the oaktag 
with the textured stimulus being longer on each trial; the $\mathrm{S}$ was to pick the longer stimulus. For Group 2 coarse tex tured stimuli were compared to the oaktag paper with the textured stimuli being shorter on each trial; the $S$ was to pick the shorter stimulus. The other two experimental groups received pretraining with the stimuli arranged such that the bias attributable to texture was in a direction opposite to the objective length differences. For Group 3 fine textured stimuli were compared to the oaktag papers with the textured stimuli being shorter on each trial; the $S$ was to pick the shorter stimulus. For Group 4 coarse textured stimuli were compared to the oaktag paper with the textured stimuli being longer; the $\mathrm{S}$ was to pick the longer stimulus.

The experimental Ss, after the initial discrimination training, and the control Ss, with no pretraining, received 20 discrimination trials with a fine-textured (320) and a coarse-textured (50) stimulus. The stimulus lengths for these trials were $7,7-1 / 16,7-2 / 16$, and $7-3 / 16$ in. On eight of the trials there was no objective length difference between the stimuli, on four trials the stimuli differed by $1 / 16$ in., on four trials they differed by $2 / 16 \mathrm{in}$. and on four trials they differed by $3 / 16$ in. On half of the latter 12 trials the fine-textured stimulus was longer and on the other half the coarse-textured stimulus was longer. The experimental Ss continued to pick the same stimulus as in the pretraining, i.e., either the longer or the shorter stimulus. On these 20 trials no information feedback was given to the Ss. The Ss were blindfolded throughout the task.

\section{Results}

Two questions were asked: (a) Were there any differences in performance during pretraining for the four experimental groups, and (b) were there any differences between the five groups in the ability to accurately discriminate length during the subsequent discrimination trials?

An analysis of variance was performed on the number of correct discriminations during pretraining for the four experimental groups. This analysis revealed that the four groups did differ $(F=6.17, \mathrm{df}=3 / 36, \mathrm{p}<.005)$. Further analysis of this finding by the Newman-Keuls test for ordered means revealed that Group 2 (coarse texture/shorter; S picking shorter) performed significantly better than Group 4 (coarse tex ture/longer; $S$ picking longer $)\left(q^{4}=5.77, \mathrm{df}=36, \mathrm{p}<.01\right)$ and Group 1 (fine texture/longer; $S$ picking longer) $\left(q^{3}=4.29\right.$, $\mathrm{df}=36, \mathrm{p}<.05)$. Where coarse texture was involved, discrimination was facilitated under conditions where facilitation was predicted and discrimination was hindered under conditions where interference was predicted. As in Experiment 1 it was again found that the predictions involving the coarse texture were confirmed while those for the fine sandpaper were not.

of the 20 discrimination trials, 12 involved real length differences. An analysis of variance was performed on the number of correct discriminations on these trials for the five groups. No significant difference was found $(F<1)$.

For the eight trials on which there were no objective differences between stimuli the S's performance was scored in terms of how well it conformed to the hypothesized biasing effect of texture, i.e., picking the finer texture as longer or the coarser texture as shorter. The dependent measure was the number of responses favoring the hypothesis minus the number of responses against the hypothesis; no preference equals 0 . An analysis of variance performed on the scores of the five groups for these eight trials found no significant differences between groups $(F=1.91$, df $=4 / 45, p>.10)$. However, further analysis revealed that the overall mean (1.24) was significantly different from chance $(0)$ in the predicted direction $(t=6.59$, $d f=45, p<.001)$. For the individual groups, three (Groups 2, 3, and Control) differed from chance at the $p<.01$ level of significance, one (Group 4) at the $\mathrm{p}<.025$ level of significance, and one (Group 1) fell just short of the $p<.05$ level of significance. Although the groups did not differ significantly from one another in the degree to which they conformed to the predictions, all groups did perform as predicted from the curvilinear hypothesis.

Thus, in general, the preliminary training for the experimental Ss had no measurable effect upon their subsequent performance in the 20 discrimination trials. However, as found in Experiment 1, texture did have a predictable effect upon length discrimination, fine texture making a given length appear longer than it objectively was and coarse texture making a given length appear shorter than it objectively was.

\section{DISCUSSION}

The results from these two experiments have substantially supported the hypothesized curvilinear relationship between texture and perceived length; as texture goes from fine to coarse, length is first overestimated and then underestimated. These experiments were less able to demonstrate that these biases could interfere with and facilitate length discrimination or could be modified through a simple training procedure. Even though Experiment 1 did not find facilitation of length discrimination, as compared to discrimination ability with untextured stimuli, the between-texture comparisons were almost all as had been predicted. Furthermore, where interference with discrimination was predicted, it was almost always found.

Experiment 2 attempted to modify the biasing effect of texture by giving Ss preliminary training of different types. Although these training procedures did not differentially affect the Ss' discrimination ability, the mean performance of the different groups and all groups taken together continued to demonstrate the biasing effect of texture.

It should be pointed out that in making their judgments Ss obtained proprioceptive and kinesthetic information about the true length of the stimuli. The consistent but small bias of length judgment by texture might be enhanced if this source of valid information were eliminated. A possible procedure to eliminate proprioceptive and kinesthetic information would be to have $\mathrm{E}$ move the texture lengths across S's passive finger.

One observation concerning the S's response to this type of situation would be instructive for future studies. Some Ss find the task in these experiments to be realistic and it was clear that they were feeling and reporting what they perceived to be real length differences. Other Ss, particularly in Experiment 2, found the task to be somewhat unrealistic. It was clear from some of their attitudes and actual statements that they had extreme difficulty in feeling any differences in length even in situations where the length difference should have been well above threshold. It seems possible that there are fairly large individual differences in the ability to attend to or discretely experience tactual information. If future experiments controlled for these individual differences, it is possible that the biasing effect of the different textures would interact with length discrimination ability more predictably. It would also be quite interesting to examine these same phenomena with a population of unsighted or partially sighted Ss for whom tactual information is ecologically more salient.

It has been mentioned that several investigators have used differences in texture to present information to the blind, e.g., information that is usually conveyed by graphs, charts, and diagrams. The results of the present experiments suggest that when information is conveyed by differences in length, as in bar graphs, the longer elements should be made of fine texture and the shorter elements of coarse tex ture.

While the effect of texture upon perceived length is not as overwhelming a perceptual phenomenon as some of the visual 
illusions, it is suggested that the differences attributable to texture might be utilized when teaching the blind certain coding systems. For example, if one wanted to develop a coding system to represent differences in depth or distance, the phenomena described here suggest that the blind be taught that coarse texture means near distance while fine texture means far away. Although it cannot be suggested that this type of code is inherent in the human organism, it is suggested that this type of code might be easier to teach than some other arbitrary code.

\section{REFERENCES}

LINDQUIST, E. F. Design and analysis of experiments in psychology and education. Boston: Houghton Mifflin, 1953.

NOLAN, C. Y., \& MORRIS, J. E. Further results in the development of a test of roughness discrimination. International Journal of Education for the Blind, 1960, 10, 48-50.

SCHIFF, W., KANFER, L., \& MOSAK, S. Informative tactile stimuli in the perception of direction. Perceptual \& Motor Skills, 1966, 23, 1315-1355.

SHERMAN, J. C. Needs and resources in maps for the blind. New Outlook for the Blind, 1965, 59, 130-134.

STEVENS, S. S., \& HARRIS, J. R. The scaling of subjective roughness and smoothness. Journal of Experimental Psychology, 1962, 64, 489-494.

WINER, B. J. Statistical principles in experimental design. New York: McGraw-Hill, 1962.

WOODWORTH, R. S., \& SCHLOSBERG, H. Experimental psychology. (Rev. ed.) New York: Henry Holt, 1960.

\section{NOTES}

1. This research was supported by N.I.H. Grant MH 07631 to the University of Minnesota. The authors wish to thank Meredeth Fink and Rosanne Aldridge for their assistance in Experiment ?.

2. Address: Psychology Department, University of Georgia, Athens, Georgia 30601.

3. Lomov, B. F., \& Vekker, L. M. Personal communication, 1960. (Accepted for publication January 6, 1969.) 